\title{
SPECTRA OF INFINITE GRAPHS: TWO METHODS OF COMPUTATION
}

\author{
LEONID GOLINSKII
}

Abstract. Two methods for computation of the spectra of certain infinite graphs are suggested. The first one can be viewed as a reversed Gram-Schmidt orthogonalization procedure. It relies heavily on the spectral theory of Jacobi matrices. The second method is related to the Schur complement for block matrices. A number of examples including finite graphs with tails, chains of cycles and ladders are worked out in detail.

Mathematics subject classification (2020): Primary 05C63; Secondary 05C76, 47B36, 47A10, 47B15. Keywords and phrases: Adjacency operator, Jacobi matrices of finite rank, Jost function, block matrix, right limit, Schur complement.

\section{REFERENCES}

[1] R. B. BAPAT, Graphs and Matrices, Springer, Universitext, 2011.

[2] A. Böttcher and B. Silbermann, Introduction to Large Truncated Toeplitz Matrices, Springer, 1999.

[3] J. BREUER, Singular continuous spectrum for the Laplacian on certain sparse trees, Comm. Math. Phys., 269 (2007), 851-857.

[4] J. BREUER, Singular continuous and dense point spectrum fr sparse trees with finite dimension, in "Probability and Mathematical Physics", v. 47 (2007), 65-83.

[5] A. E. Brouwer, W. H. Haemers, Spectra of Graphs, Springer, Universitext, 2012.

[6] F. CHung, Spectral graph theory, volume 92 of CBMS Regional Conference Series in Mathematics. Published for the Conference Board of the Mathematical Sciences, Washington, DC, 1997.

[7] D. M. Cvetković, M. Doob, H. SACHs, Spectra of Graphs - Theory and Applications, Academic Press, 1980.

[8] D. Damanik, B. Simon, Jost function, and Jost solutions for Jacobi matrices, II. Decay and analyticity, Int. Math. Res. Notes (2006), art. ID 19396, 1-32.

[9] F. Fidaleo, D. Guido, And T. Isola, Bose-Einstein condensation on inhomogeneous amenable graphs, in: "Infinite Dimensional Analysis, Quantum Probability and Related Topics", 14 (2011), no. 2, 149-197.

[10] F. Fidaleo, Harmonic Analysis on Inhomogeneous Amenable Networks and the Bose-Einstein Condensation, Journal of Stat. Phys., 160 (2015), no. 3, 715-759.

[11] I. C. Gokhberg, M. G. Krein, Introduction to the Theory of Linear Nonselfadjoint Operators, AMS, Providence, RI, 1969.

[12] L. GolinskiI, Spectra of infinite graphs with tails, Linear and Multilinear Algebra, 64 (2016), no. 11, 2270-2296.

[13] L. Golins KII, Spectra of infinite graphs via Schur complement, Operators and Matrices, 11 (2017), no. 2, 389-396.

[14] L. GOLInSKII, Spectra of comb graphs with tails, preprint: arxive1904:06678, 2019.

[15] R. Horn And C. Johnson, Matrix Analysis, CUP, Cambridge, 1986.

[16] R. Killip, B. Simon, Sum rules for Jacobi matrices and their applications to spectral theory, Ann. Math., 158 (2003), 253-321.

[17] R. KozHAn, Finite range perturbations of finite gap Jacobi and CMV operators, Advances in Math., 301 (2016), 204-226. 
[18] R. Kozhan, On Gaussian random matrices coupled to the discrete Laplacian, to appear in Oper. Theory Adv. Appl. (2019), Issue "Analysis as a Tool in Mathematical Physics: in memory of Boris Pavlov" (editors P. Kurasov, A. Laptev, S. Naboko, and B. Simon).

[19] Y. LAST AND B. SimON, The essential spectrum of Schrödinger, Jacobi, and CMV operators, J. Anal. Math., 98 (2006), 183-220.

[20] V. LEBID, Spectral analysis of a double star graph with infinite rays, Proc. of Math. Institute of NANU, 11 (2014), no. 3, 166-172.

[21] V. Lebid, L. Nizhnik, Spectral analysis of locally finite graphs with one infinite chain, Proc. Ukranian Academy of Sci., (2014), no. 3, 29-35.

[22] V. Lebid, L. NizHniK, Spectral analysis of certain graphs with infinite chains, Ukr. J. Math. 66 (2014), no. 9, 1193-1204.

[23] X. LiU, S. ZHou, Spectral characterizations of propeller graphs, Electron J. Linear Algebra 27 (2014), 19-38.

[24] B. MohaR, The spectrum of an infinite graph, Linear Alg. Appl., 48 (1982), 245-256.

[25] B. MohaR, W. Woess, A survey on spectra of infinite graphs, Bull. London Math. Soc., 21 (1989), 209-234.

[26] L. P. Nizhnik, Spectral analysis of metric graphs with infinite rays, Methods of Func. Anal. and Topology, 20 (2014), 391-396.

[27] G. Pólya AND G. SZEgő, Problems and Theorems in Analysis, v. II, Springer, 1998.

[28] C. Remling, The absolutely continuous spectrum of Jacobi matrices, Ann. of Math., 174 (2011), $125-171$.

[29] I. SCHUR, Über Potenzreihen, die im Innern des Einheitskreises beschränkt sind, I, J. Reine Angew. Math., 147 (1917), 205-232.

[30] A. SCHWEnK, Computing the characteristic polynomial of a graph, Graphs and combinatorics. Lect. Notes Math. 406 (1974), 153-172.

[31] B. Simon, Operators with singular continuous spectrum, VI. Graph Laplacians and Laplace-Beltrami operators, Proc. Amer. Mat. Soc., 124 (1996), no.4, 1177-1182.

[32] B. Simon, Orthogonal polynomials on the unit circle. Part 2: Spectral Theory, Colloquium Publications, v. 54, AMS, Providence, RI, 2005.

[33] B. Simon, Szegó's Theorem and its Descendants, Princeton Uiversity Press, 2011.

[34] B. Simon And G. Stolz, Operators with singular continuous spectrum, V. Sparse potentials, Proc. Amer. Math. Soc., 124 (1996), 2073-2080.

[35] G. TeSCHL, Jacobi operators and completely integrable nonlinear lattices, Mathematical Surveys and Monographs, v. 72, AMS, Providence, RI, 2000. 